\title{
COMBINED INSTRUMENTS FOR THE SCREENING OF DEMENTIA IN OLDER PEOPLE WITH LOW EDUCATION
}

\author{
Cássio M.C. Bottino', Sonia E. Zevallos-Bustamante', Marcos A. Lopes ${ }^{1,2}$, \\ Dionisio Azevedo', Sérgio R. Hototian', Wilson Jacob-Filho ${ }^{3}$, Julio Litvoc ${ }^{4}$
}

\begin{abstract}
Objective: To determine which combination of cognitive tests and informant reports can improve the diagnostic accuracy of dementia screening in low educated older people. Method: Patients with mild to moderate dementia ( $n=34$ ) according to ICD-10 and DSM-III-R criteria and 59 older controls were assessed with the Mini-Mental State Examination (MMSE) and the Fuld Object Memory Evaluation (FOME). Informants were assessed using the Informant Questionnaire on Cognitive Decline in the Elderly and the Bayer-Activities of Daily Living Scale. Results: The 4 instruments combined with the mixed rule correctly classified $100 \%$ and the logistic regression (weighted sum) classified $95.7 \%$ of subjects. The weighted sum had a significantly larger ROC area compared to MMSE $(p=0.008)$ and FOME $(p=0.023)$. The specificity of the tested combinations was superior to the MMSE alone ( $p=0.002$ ). Conclusions: Cognitive tests combined with informant reports can improve the screening of mild to moderate dementia in low educated older people.
\end{abstract}

KEY WORDS: dementia, screening, cognitive tests, informant report.

\section{Instrumentos combinados para o rastreio de demência em idosos com baixo nível educacional}

Resumo - Objetivo: Determinar qual combinação de testes cognitivos e avaliações do informante pode melhorar o rastreio de demência em idosos com baixo nível educacional. Método: Pacientes com demência leve a moderada ( $n=34$ ) de acordo com critérios da CID-10 e DSM-III-R, e 59 controles idosos foram avaliados com o Mini-Exame do Estado Mental (MEEM) e com o "Fuld Object Memory Evaluation" (FOME). Informantes foram avaliados com o "Informant Questionnaire on Cognitive Decline in the Elderly" e a escala Bayer-Atividades da Vida Diária. Resultados: Os quatro instrumentos combinados com a regra mista classificaram $100 \%$ e a regressão logística (soma ponderada) classificou $95,7 \%$ dos sujeitos. A soma ponderada teve uma área da curva ROC significativamente maior comparada ao MEEM ( $p=0,008)$ e FOME ( $p=0,023)$. A especificidade das combinações testadas foi superior ao MEEM isolado $(p=0,002)$. Conclusões: Testes cognitivos combinados com relatos dos informantes podem melhorar o rastreio de demência leve a moderada em idosos com baixo nivel educacional.

PALAVRAS-CHAVE: demência, rastreio, testes cognitivos, relato de informantes.

The development of effective treatment strategies for dementia and specially for Alzheimer's disease that can delay disease progression speed and improve patients' and relatives' quality of life have stimulated clinicians and researchers to search for earlier and more precise diagnoses.

The use of combined tests has shown promising results for increasing the accuracy of dementia screening.
A valuable strategy seems to be the combination of two short cognitive tests ${ }^{2}$ that achieved $100 \%$ of sensitivity and specificity for moderate to severe dementia patients and a higher efficacy compared to either test alone (Abbreviated Mental Test Score - AMTS; Mental Status Questionnaire; Mini-Mental State Examination - MMSE; and Short Portable Mental Status Questionnaire). Other strategy studied was the combination of a cognitive test as the

\footnotetext{
'Projeto Terceira Idade (PROTER), Instituto e Departamento de Psiquiatria, Faculdade de Medicina da Universidade de São Paulo, São Paulo SP, Brazil; ${ }^{2}$ Hospital das Clínicas da Faculdade de Medicina de Ribeirão Preto, Universidade de São Paulo, Ribeirão Preto SP, Brazil; ${ }^{3}$ Departamento de Clínica Médica, Faculdade de Medicina da Universidade de São Paulo, São Paulo SP, Brazil; ${ }^{4}$ Departamento de Medicina Preventiva, Faculdade de Medicina da Universidade de São Paulo, São Paulo SP, Brazil. Financing: Fundação de Apoio à Pesquisa do Estado de São Paulo (FAPESP), Proc. No. 01/05959-7.
}

Received 20 October 2008, received in final form 18 December 2008. Accepted 11 March 2009.

Dr. Cássio M.C. Bottino - Instituto de Psiquiatria do Hospital das Clínicas da FMUSP - Rua Dr. Ovídio Pires de Campos 785 - 05403-010 São Paulo SP Brasil.E-mail: cbottino@usp.br 
MMSE with an informant report as the Informant Questionnaire of Cognitive Decline in the Elderly (IQCODE) resulting in more accurate prediction of dementia cases than either test alone 3 . Preliminary results of our group ${ }^{4}$, assessing 30 mild to moderate patients with dementia and 46 elderly controls with the MMSE, and the informants with the IQCODE and the Bayer-Activities of Daily Living Scale (B-ADL) showed that the accuracy of the MMSE alone was $86.8 \%$. On the other hand, combining these instruments with the "And" rule classified correctly $92.1 \%$ of the subjects, suggesting that this strategy can improve the detection of mild to moderate cases of dementia.

Comparing the utility of 8 tests and scales for the diagnosis of dementia in 152 elderly subjects, the authors ${ }^{5}$ found that age, gender and basic tests (the AMDP scale - a clinician judgment of autonomy or dependence, the Clock Drawing test and the MMSE) had a superior diagnostic value compared to sophisticated neuropsychological for the diagnostic work-up of dementia.

Another very important issue regarding the screening of dementia is the influence of schooling and cultural differences on some universally applied tests like the $\mathrm{MMSE}^{6}$, as reported in the northeast of Brazil by BritoMarques and Cabral-Filho ${ }^{7}$. In many developing countries or evaluating minority populations in developed countries, the educational bias can limit or jeopardize the use of some tests.

In the present study, two cognitive tests applied to the subjects, the MMSE ${ }^{6,8}$, and the Fuld Object Memory Evaluation (FOME) ${ }^{9}$, associated to two scales applied to an informant, the IQCODE ${ }^{10}$ and the Bayer Activities of Daily Living Scale (B-ADL) $)^{11,12}$ were tested. The objective was to investigate which combination of these instruments could have adequate sensitivity and specificity for the screening of mild to moderate dementia even in low educated older people.

\section{METHOD}

\section{Participants}

Thirty-four patients with mild to moderate dementia according to the International Classification of Mental and Behavioural Disorders (ICD-10) ${ }^{13}$ and the Diagnostic and Statistical Manual of Mental Disorders, $3^{\text {rd }}$ edition, revised (DSM-III-R) ${ }^{14}$ criteria, and 59 older controls were evaluated. Dementia cases were recruited from the ambulatory of the Old Age Research Group (PROTER), followed for at least 6 months, and evaluated according to a standardized work-up for dementia, including a computerized tomography or magnetic resonance imaging. An experienced geriatric psychiatrist reviewed the patients' files and re-evaluated both patients and informants before testing.

Elderly controls were recruited among patients' family members and patients from the Geriatric Medicine ambulatory at the same institution. Controls were previously evaluated by psychia- trists with Brazilian versions of a psychiatric screening questionnaire (SRQ-20) $)^{15}$ and one cognitive screening test (AMTS) ${ }^{16}$.

Subjects were divided in 3 groups by socio-economic status and educational level (high, medium and low). Patients and informants were evaluated by interviewers blind to clinical status.

All the subjects and relatives signed a written informed consent and the study was previously approved by the research ethics committee of HCFMUSP (nr. 698/00).

\section{Instruments}

The MMSE ${ }^{6,8}$ assesses five areas of cognition labelled as "orientation", "registration", "attention and calculation", "recall", and "language". Scores of $23 / 24$ points or less out of a maximum of 30 have been recognized as indicating cognitive deficits and possible dementia ${ }^{17}$.

The FOME was designed to reduce cultural and educational bias and evaluates learning and recall, tactile recognition, leftright discrimination and verbal fluency. The test showed fairly good positive and negative predictive values for dementia in a community sample ${ }^{18}$. After the visual inspection, the subjects were asked to identify and name ten common objects (key, comb, cup, ball) placed in a bag, followed by the the selective recall of them ( 5 times) with verbal fluency tasks (4 times) as distractors. The delayed recall task is performed after 15 minutes 9 . In the present study, only the sum of all correct answers given by the subjects in the 5 recall trials (FOME total) was presented.

The IQCODE is an instrument designed to measure the cognitive decline, through the interview of a caregiver or a close relative. A review pointed out that the IQCODE has high reliability and is relatively unaffected by education, pre-morbid ability or by proficiency in the dominant language ${ }^{19}$. The questionnaire comprises 26 questions, asking about the performance of the patient in different everyday situations compared to 10 years before. Scores range from 26 to 130 points and the questionnaire takes 10 minutes to be administered ${ }^{10}$.

The Bayer-Activities of Daily Living Scale (B-ADL) assesses the frequency of the patients' difficulties to perform his or her everyday activities. The B-ADL was designed to be applied in several cultures, for its sensitivity to detect mild cases of dementia and for measuring different functional aspects of the individual ${ }^{12,20}$. It is a 10-point scale, with 25 questions which are completed by a caregiver or a close relative"1.

The socio-economic classification was made according to the Brazilian Association of Market Research Scale ${ }^{21}$.

\section{Statistical analysis}

The comparison between patients and controls was made with Pearson Chi-square and Student $t$ test for categorical and continuous variables respectively. The groups of patients and healthy elderly subjects divided according their social classes were compared through one-way ANOVA, with post-hoc Bonferroni Correction for multiple testing, or Kruskal-Wallis test when appropriate. 
Table 1. Socio-demographic characteristics.

\begin{tabular}{llccc}
\hline & & \multicolumn{2}{c}{ Diagnosis } & \\
\cline { 3 - 4 } & & Dementia & Control & Test and p value \\
\hline \multirow{2}{*}{ Gender } & $M$ & $16(47.1 \%)$ & $12(20.3 \%)$ & $\chi^{2}=7.31$ \\
\multirow{3}{*}{ Age } & $\mathrm{F}$ & $18(52.9 \%)$ & $47(79.7 \%)$ & $\mathrm{p}=0.007$ \\
\multirow{3}{*}{ Education } & Mean & 73.70 & 69.25 & $\mathrm{t}=3.75$ \\
& $(\mathrm{SD})$ & $(5.8)$ & $(5.3)$ & $\mathrm{p}<0.001$ \\
\multirow{2}{*}{ Social class } & Mean & 5.44 & 4.91 & $\mathrm{t}=0.62$ \\
& (SD) & $(4.0)$ & $(3.8)$ & $\mathrm{p}=0.53$ \\
& High & $11(32.4 \%)$ & $20(33.9 \%)$ & \\
& Medium & $13(38.2 \%)$ & $19(32.2 \%)$ & $\chi^{2}=0.37$ \\
& Low & $10(29.4 \%)$ & $20(33.9 \%)$ & $\mathrm{p}=0.82$ \\
\hline
\end{tabular}

$\chi^{2}$ : Pearson Chi-square; t: Student $t$ test.

Table 2. Age, education and instruments' means according to social class.

\begin{tabular}{|c|c|c|c|c|c|c|c|c|}
\hline \multirow{2}{*}{$\begin{array}{l}\text { Variable's } \\
\text { means (SD) }\end{array}$} & \multicolumn{3}{|c|}{ Dementia } & \multirow{2}{*}{$\begin{array}{l}\text { Test and } \\
\mathrm{p} \text { value }\end{array}$} & \multicolumn{3}{|c|}{ Controls } & \multirow{2}{*}{$\begin{array}{l}\text { Test and } \\
\mathrm{p} \text { value }\end{array}$} \\
\hline & High & Medium & Low & & High & Medium & Low & \\
\hline Age & $71.8(4.9)$ & $76.0(6.6)$ & $72.7(4.8)$ & $\begin{array}{c}F_{2.31}=1.90 \\
p=0.16\end{array}$ & $68.0(5.1)$ & $69.4(5.7)$ & $70.3(5.0)$ & $\begin{array}{c}F_{2.56}=0.90 \\
p=0.40\end{array}$ \\
\hline Education & $7.9(5.5)$ & $4.8(2.8)$ & $3.5(1.5)$ & $\begin{array}{c}\mathrm{KW}_{2}=4.58 \\
\mathrm{p}=0.10\end{array}$ & $6.5(4.6)$ & $4.5(3.5)$ & $3.7(2.4)$ & $\begin{array}{c}\mathrm{KW}_{2}=3.82 \\
\mathrm{p}=0.14\end{array}$ \\
\hline MMSE & $21.7(5.2)$ & $19.8(6.3)$ & 17.7 (6.1) & $\begin{array}{c}F_{2.31}=1.19 \\
p=0.31\end{array}$ & $28.7^{*}(1.3)$ & $27.9(2.1)$ & $27.1(2.0)$ & $\begin{array}{c}F_{2.56}=3.59 \\
p=0.03\end{array}$ \\
\hline FOME & 19.4 (15.0) & $18.6(10.2)$ & 16.5 (11.5) & $\begin{array}{c}F_{2.31}=0.15 \\
p=0.85\end{array}$ & $39.8(5.0)$ & 41.1 (5.9) & $43.0(3.9)$ & $\begin{array}{c}F_{2.56}=2.03 \\
p=0.14\end{array}$ \\
\hline IQCODE & $4.1(0.2)$ & $4.0(0.5)$ & $4.1(0.6)$ & $\begin{array}{c}F_{2.31}=0.09 \\
p=0.91\end{array}$ & $3.1(0.5)$ & $3.1(0.1)$ & $2.7(0.5)$ & $\begin{array}{c}F_{2.56}=3.46 \\
p=0.03\end{array}$ \\
\hline B-ADL & $6.0(2.3)$ & $5.9(2.3)$ & 7.5 (1.7) & $\begin{array}{c}F_{2.31}=1.87 \\
p=0.17\end{array}$ & $1.8(0.9)$ & $1.5(0.7)$ & $1.6(0.6)$ & $\begin{array}{c}F_{2.56}=0.74 \\
p=0.48\end{array}$ \\
\hline
\end{tabular}

F: One-way ANOVA; KW: Kruskal Wallis test; *MMSE controls - High vs. Low: $p=0.029$

The instruments individual cut off points were chosen with the receiver operating characteristic curve (ROC) analysis. The instruments were combined with a compensatory rule ("Or" rule), with a conjunctive rule ("And"), with a mixed rule (any cognitive test altered "And" any functional scale altered), and with the logistic regression analysis to combine the four instruments together (weighted sum).

Binomial two-tailed tests were used for the comparison of the sensitivity and specificity values of individual and combined screening instruments. The positive predictive values (PPV) and negative predictive values (NPV) were corrected for a dementia prevalence rate of $7 \%$, reported previously in Brazil ${ }^{22}$.

\section{RESULTS}

The demented patients were older (mean=73.7 years, $S D=5.8)$ and with an excess of women compared to the healthy controls. Nineteen $(55.9 \%)$ patients were classified as mildly and 15 (44.1\%) as moderately demented, according to the DSM-III-R. Regarding schooling, $69 \%$ of the sam- ple had studied less than 4 years. Both groups could be considered low educated (mean $=5.10$ years, SD=3.86) (Table 1). The prevalence of dementia in the sample was $36.6 \%$.

In Table 2, a comparison between patients and healthy controls divided according their socio-economic status showed a statically significant difference for the MMSE scores of the control group.

The areas under the ROC Curves were: $M M S E=0.941$; FOME=0.957; IQCODE=0.958; B-ADL=0.975; and weighted sum $=0.997$. The performance of the 4 instruments were similar with a small advantage of the B-ADL over the other 3 instruments (non significant) (Figure). The weighted sum had a significantly larger area compared to the MMSE $(p=0.008)$ and the FOME ( $p=0.023)$.

In Table 3, the performance of the individual tests was compared to combinations using the "And" rule, the mixed rule and the weighted sum. On the pairwise comparisons, combinations using only the informant reports were tested but were not presented as the approaches combin- 


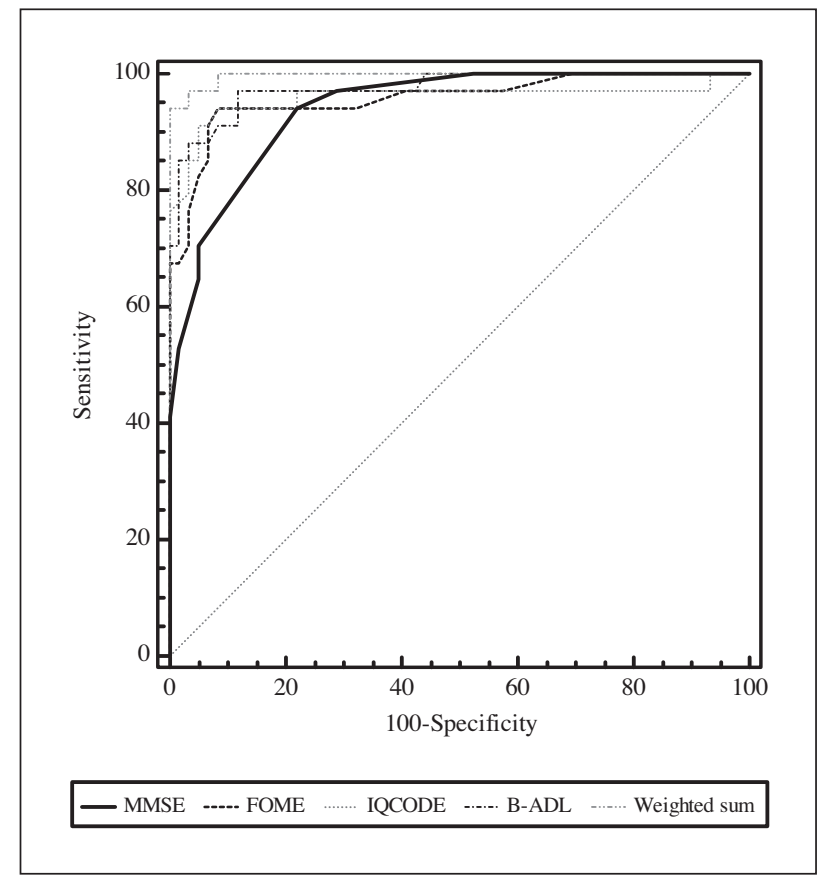

Figure. ROC Curves' comparison.

ing one cognitive test and one informant report provided better results. Combinations using the "Or" rule were also tested but were not presented as the "And" rule provided better results.

All the combinations ("And" rule, mixed rule and weighted sum) were superior to the MMSE alone regarding the specificity $(p<0.002)$, but not the sensitivity values. The 4 instruments combined through the logistic regression achieved an accuracy of $95.7 \%$ (sensitivity $94.1 \%$ and specificity $96.6 \%)$. The 4 instruments combined using the mixed rule correctly classified $100 \%$ of subjects (sensitivity and specificity $100 \%$ ).

\section{DISCUSSION}

The combination of tests and scales to increase the accuracy of dementia screening was reported before with encouraging results ${ }^{2-5}$. However, the influence of low schooling on tests' performance can compromise these results or make them less consistent. In the present study, even those subjects classified with high socio-economic status had at best a mean of 8 years of schooling, and $69 \%$ of the sample had 4 or less years of schooling, what can be probably found in many developing countries as Brazil or in minority population samples in developed countries. The combinations of instruments, especially those combining one cognitive test (MMSE or FOME) to one informant report (IQCODE or B-ADL) improved the utility of the instruments alone, using the MMSE as a reference, considering the specificity values. The complementary approach of cognitive tests and informant reports can probably explain the results found. Considering the pairwise comparisons, the combination using FOME "And" IQCODE provided a higher sensitivity value, what can be explained because the FOME is less influenced by schooling than the MMSE and the IQCODE can capture the cognitive decline during a period of time, while the B-ADL evaluates current functional deficits.

The 4 instruments combined with the logistic regression (weighted sum) had a significantly larger area on the ROC curve analysis compared to the MMSE, the FOME, and the IQCODE. Combining the logical operators through the mixed rule resulted in a perfect differentiation between patients and controls. Both approaches (mixed rule

Table 3. Comparison of the instruments and combinations.

\begin{tabular}{|c|c|c|c|c|c|}
\hline Instruments & Sen $\%$ & Spe \% & Acur \% & $\mathrm{PPVc} \%$ & NPVc \% \\
\hline MMSE $\leq 26$ & 94.1 & 78.0 & 83.8 & 24.4 & 99.4 \\
\hline FOME $\leq 34$ & 94.1 & 91.5 & 92.4 & 45.5 & 99.5 \\
\hline IQCODE $\geq 3.40$ & 94.1 & 91.5 & 92.4 & 45.5 & 99.5 \\
\hline B-ADL $\geq 3.19$ & 88.2 & 96.6 & 93.5 & 66.2 & 99.1 \\
\hline MMSE $\leq 26$ “And" FOME $\leq 34$ & 88.2 & 98.3 & 94.6 & 79.6 & 99.1 \\
\hline MMSE $\leq 26$ “And” B-ADL $\geq 3.19$ & 85.3 & 100.0 & 94.6 & 100.0 & 98.8 \\
\hline MMSE $\leq 26$ "And" IQCODE $\geq 3.40$ & 88.2 & 100.0 & 95.7 & 100.0 & 99.1 \\
\hline FOME $\leq 34$ “And" B-ADL $\geq 3.19$ & 88.2 & 100.0 & 95.7 & 100.0 & 99.1 \\
\hline FOME $\leq 34$ "And" IQCODE $\geq 3.40$ & 98.3 & 100.0 & 93.5 & 100.0 & 99.9 \\
\hline Logistic regression - 4 Instruments & 94.1 & 96.6 & 95.7 & 67.6 & 99.5 \\
\hline Mixed Rule & 100.0 & 100.0 & 100.0 & 100.0 & 100.0 \\
\hline \multicolumn{6}{|l|}{ MMSE $\leq 26$ "Or " FOME $\leq 34$ "And" } \\
\hline IQCODE $\geq 3.40$ "Or" B-ADL $\geq 3.19$ & & & & & \\
\hline
\end{tabular}

Sen: Sensitivity; Spe: Specificity; Acur: Acuracy; PPVc: Positive predictive value corrected for a 7\% dementia prevalence; NPVc: Negative predictive value corrected for a $7 \%$ dementia prevalence. 
and weighted sum) were superior to the MMSE alone regarding the specificity, but not the sensitivity. Regarding the positive and negative predictive values, corrected for the rate of dementia prevalence in community samples, a better performance of the combinations tested is more apparent, with the only exception being the good results found with the B-ADL scale alone.

The validity of a complete (S-IQCODE) and a shortened (SS- IQCODE) Spanish version of the IQCODE for the diagnosis of dementia were studied before in a clinical setting ${ }^{23}$. Thirty-eight outpatients $(71 \%)$ were diagnosed as demented and the best results were obtained with the combination of the MMSE and S-IQCODE with the "And" rule. ${ }^{23}$ The sample evaluated ${ }^{21}$ was comparable to ours, regarding schooling, but we found higher values for the combinations tested ("And" rule, weighted sum, and mixed rule) considering the specificity, negative predictive values and accuracy. The choice of cut-off points could probably explain those differences for the "And" rule, but the results found in the present study with the weighted sum and the mixed rule suggest that these strategies can be helpful to increase the accuracy of dementia screening.

Mackinnon and Muligan ${ }^{3}$ assessed 106 elderly hospital or outpatients with the MMSE and the IQCODE combined through the logistic regression and the "Or" rule. Both strategies improved performance over that of either test used alone. The authors reported that with the "Or" rule, sensitivity was $93 \%$ and specificity was $81 \%$, and with the logistic regression, combining both instruments, sensitivity was $97 \%$ and specificity was $85 \%$ for demen$\mathrm{tia}^{3}$. We found higher values for the combinations tested ("And" rule, weighted sum, and mixed rule) considering the specificity, positive and negative predictive values. As reported by Mackinnon and Muligan 3 , using the "And" rule in the present study, caused an improvement in specificity and a decrease in sensitivity compared to the MMSE alone, but there was also an increase in accuracy and in the positive predictive values for most of the combinations tested. However, perusing at Table 3, the combinations FOME "And" IQCODE, and the mixed rule provided higher values regarding not only the sensitivity, but also for the other indices. Probably, because of the high sensitivity value for the MMSE alone, the use of the "Or" rule did not have a positive impact in our sample.

In other study, Mackinnon et al. ${ }^{24}$ evaluated 646 community older subjects with the MMSE and the 16-item form of the IQCODE. Dementia was diagnosed according to DSM-III-R criteria and $11 \%$ of the sample had left school under the age of 14 years. Confirming previous results ${ }^{3}$, the combination of the IQCODE and the MMSE with the "Or" rule and with the weighted sum were superior to other methods of combination, improving screening accuracy. The principal difference between the studies was the cut off points that yielded a better performance in the community sample, which had a lower prevalence of dementia than the clinical sample (5.6\% versus $54.7 \%$ ). Compared with our results, obtained with a sample with lower schooling and intermediate or equivalent (if corrected) dementia prevalence rates $(36.6 \%$, or $7 \%$ corrected), the cut off points using the same instruments were quite similar (MMSE $\leq 26$ "And" IQCODE $\geq 3.4$ ), but applied to our sample with a conjunctive ("And") rule enhanced specificity.

Investigating the same strategy (combining MMSE and IQCODE), 323 patients were assessed at a memory clinic ${ }^{25}$. The sample had a high prevalence of dementia (70.9\%), and $31 \%$ had left school at the age of 16 and older. Logistic regression analysis showed that the combination of the MMSE and the IQCODE produced a slightly more accurate prediction of dementia than either test used alone. However, combining the MMSE and the IQCODE with the logistic regression did not result in any improvement over the use of the MMSE alone, contrasting with the previous studies presented. The authors discussed that perhaps in samples with lower dementia prevalence rates as in general practice (or in community samples) the weighted sum might be of greater utility ${ }^{25}$, as we found in the present report.

Possible weakness of the present report should be cited as the relatively small sample size and the convenience sample evaluated (selected ambulatory patients with mild to moderate dementia and highly motivated geriatric controls) that can have inflated the sensitivity and specificity as well the predictive values found. Even considering the informants, relatives of subjects attending health services can give information of better quality leading to elevated figures than one would find in the community.

In the present sample, composed mostly by low educated older people, our results corroborate the findings of previous studies ${ }^{3,24}$ showing the advantage of combining a cognitive test to an informant report. This strategy could be easily implemented for epidemiological community studies as well as for primary health care, where a more active approach for dementia screening is needed as Lopponen et al. ${ }^{26}$ stated. Although two of the combination rules tested (the mixed rule and the weighted sum) were not easy to implement in clinical routine, the results found with the "And" rule also yielded promising results. The strategies reported in the present study should be tested in other low educated samples to further address their value to improve screening performance for dementia.

\section{REFERENCES}

1. Lanctot KL, Herrmann N, Yau KK, et al. Efficacy and safety of cholinesterase inhibitors in Alzheimer's disease: a meta-analysis. CMAJ 2003;169:557-564.

2. Hooijer C, Jonker C, Linderboom J. Cases of mild dementia in the community: Improving efficacy of case findings by con- 
current use of pairs of screening tests. Int J Geriatr Psychiatry 1993;8:561-564.

3. Mackinnon A, Mulligan R. Combining cognitive testing and informant report to increase accuracy in screening for dementia. Am J Psychiatry 1998;155:1529-1535.

4. Bustamante SEZ, Bottino CM, Lopes MA, et al. Combined instruments on the evaluation of dementia in the elderly: preliminary results. Arq Neuropsiquiatr 2003;61:601-606.

5. De Lepeleire J, Heyrman J, Baro F, Buntinx F. A combination of tests for the diagnosis of dementia had a significant diagnostic value. J Clin Epidemiol 2005;58:217-225.

6. Folstein MF, Folstein SE, McHugh PR. "Mini-mental state". A practical method for grading the cognitive state of patients for the clinician. J Psychiatr Res 1975;12:189-198.

7. Brito-Marques PR, Cabral-Filho JE. The role of education in mini-mental state examination: a study in Northeast Brazil. Arq Neuropsiquiatr 2004;62:206-211.

8. Bottino CMC, Almeida OP, Tamai S, Forlenza OV, Scalco MZ, Carvalho IAM. Entrevista estruturada para diagnóstico de transtornos mentais em idosos-CAMDEX The Cambridge examination for mental disorders of the elderly. Versão brasileira (traduzida e adaptada com autorização dos editores, Cambridge University Press), São Paulo, 1999.

9. Fuld PA, Muramoto O, Blau AD, Westbrook LE, Katzman R. Cross-cultural and multi-ethnic dementia evaluation by mental status and memory testing. Cortex 1988;24:511-519.

10. Jorm AF, Jacomb PA. The informant questionnaire on cognitive decline in the elderly (IQCODE): socio-demographic correlates, reliability, validity and some norms. Psychol Medicine 1989;19:1015-1022.

11. Hindmarch I, Lehfeld H, Jongh P, Erzigkeit H. The Bayer activities of daily living scale (B-ADL). Dement Geriatr Cogn Disord 1998;9:20-26.

12. Folquitto JC, Bustamante SE, Barros SB, et al. The Bayer: activities of daily living scale (B-ADL) in the differentiation between mild to moderate dementia and normal aging. Rev Bras Psiquiatr 2007;29:350-353.

13. Organização Mundial de Saúde. Classificação de transtornos mentais de comportamento da CID-10: descrições clínicas e diretrizes diagnósticas. Porto Alegre: Editora Artes Médicas, 1993:42-68.
14. American Psychiatric Association. Manual de diagnóstico e estatística de distúrbios mentais (Terceira Edição Revista) (DSMIII-R). São Paulo: Editora Manole, 1989:112-117.

15. Mari JJ, Williams P. A validity study of a psychiatric screening questionnaire (SRQ-20) in primary care in the city of São Paulo. Br J Psychiatry 1986;148:23-26.

16. Qureshi KN, Hodkinson HM. Evaluation of a ten-question mental test in the institutionalized elderly. Age Ageing 1974;3:152-157.

17. Tombaugh $\mathrm{T}, \mathrm{Mc}$ Intyre $\mathrm{N}$. The Mini-mental state examination: a comprehensive review. J Am Geriatr Soc 1992;40:922-935.

18. Fuld PA, Masur DM, Blau AD, Crystal H, Aronson MK. Object-memory evaluation for prospective detection of dementia in normal functioning elderly: predictive and normative data. J Clin Exp Neuropsychol 1990;12:520-528.

19. Jorm AF. The Informant Questionnaire on cognitive decline in the elderly (IQCODE): a review. Int Psychogeriatr 2004;16:275-293.

20. Erzigkeit H, Lehfeld H, Pena-Casanova J, et al. The Bayer-Activities of Daily Living Scale (B-ADL): results from a validation study in three European countries. Dement Geriatr Cogn Disord 2001;12:348-358.

21. Associação Brasileira de Anunciantes (ABIPEME). XXXI Estudos Marplan. Marplan Brasil Representações e Pesquisas Ltda: São Paulo, 1989.

22. Herrera E Jr, Caramelli P, Silveira AS, Nitrini R. Epidemiologic survey of dementia in a community-dwelling Brazilian population. Alzheimer Dis Assoc Disord 2002;16:103-108.

23. Del-Ser T, Morales JM, Barquero MS, Canton R, Bermejo F. Application of a spanish version of the "Informant Questionnaire on Cognitive Decline in the Elderly" in the clinical assessment of dementia. Alzheimer Dis Assoc Disord 1997;11:3-8.

24. Mackinnon A, Khalilian A, Jorm AF, Korten AE, Christensen $\mathrm{H}$, Mulligan R. Improving screening accuracy for dementia in a community sample by augmenting cognitive testing with informant report. J Clin Epidemiol 2003;56:358-366.

25. Knafelc R, Lo Giudice D, Harrigan S. The combination of cognitive testing and an informant questionnaire in screening for dementia. Age Ageing 2003;32:541-547.

26. Lopponen M, Raiha I, Isoaho R, Vahlberg T, Kivela SL. Diagnosing cognitive impairment and dementia in primary health care -a more active approach is needed. Age Ageing 2003;32: 606-612. 